\title{
The Potential of Laser Excitation Spectroscopy
}

The following are summaries of seven papers presented at a joint meeting of the Molecular Spectroscopy Group and the East Anglia Region, held on March 20th, 1991, at the University of East Anglia, Norwich.

\section{Introduction to Laser Excitation Spectroscopy}

\author{
David L. Andrews \\ School of Chemical Sciences, University of East Anglia, Norwich NR4 7TJ
}

The application of lasers to chemical analysis is expected to become commonplace before the end of the century. One reason is the increasing sophistication and reliability of laserbased systems. This in turn is leading to the commercial exploitation of some of the newer laser-based forms of spectroscopic analysis. The routine uses of lasers as sources for Raman studies or as ablation devices for the elemental analysis of heterogeneous solids now represent only part of modern laser spectroscopy.

The generic term 'laser excitation spectroscopy' adopted here signifies a diverse collection of techniques in which spectroscopic information is directly derived through the excitation of samples by laser radiation. Raman scattering is excluded on the grounds that it is not specifically an excitation process (as anti-Stokes lines are generated). Processes involving laser ablation are included only if lasers are involved in the spectroscopy and the vaporization of samples.

Excitation spectroscopy principally concerns techniques in which the absorption of laser light induces transitions between electronic quantum states. In addition to molecular photoabsorption spectroscopy, other important techniques in this category are laser-induced fluorescence, multiphoton absorption, laser mass spectrometry and certain ultra-fast techniques.

One can trace a few key factors in the increasing utilization of laser-based forms of analysis. Certainly when lasers first appeared they were poorly suited to absorption spectroscopy, where a source emitting either broadband or tunable output is generally required, and high intensities are mostly not too important. It was the development of tunable systems such as the dye laser which first began to open up the real potential for spectroscopic applications. The attractive combination of high monochromaticity with tunability led to such lasers gradually making inroads into spectroscopy.

This trend has recently been given a boost by the development of several new tunable solid-state lasers, such as the alexandrite laser. $^{1}$ Such lasers represent an increasingly attractive alternative to tunable dye lasers. Their chief advantages are appreciably more rugged and compact construction and operation without the use of toxic organic chemicals. At the same time the range of available diode laser wavelengths has been encroaching further into the longwavelength end of the visible region, offering new scope for high-resolution measurements in those types of electronic spectroscopy not too dependent on high power and broad tuning range.

In spite of the wide range of devices now available it is possible to identify characteristics which are distinctive of the output from laser sources. These are principally coherence, collimation, intensity and monochromaticity. In pulsed systems the generally very short pulse duration is also a significant parameter. The optics and instrumentation asso- ciated with laser systems in any given set-up naturally have a bearing on the radiation parameters actually experienced by a sample, and also frequently modify pulse and polarization characteristics.

What was at first the most widely vaunted attribute of the laser, the coherent nature of its emission, is in general the least significant for spectroscopic purposes. While there exist a number of so-called coherence spectroscopies, which are invariably studied with laser light, the nature of most is such that given an intense and well-collimated input, laser-like emission is generated in a well-defined direction and with reasonable intensity. However, similar observations would, in principle, be made using any incoherent source with the same degree of collimation and intensity.

The physically narrow beamwidth and small divergence of laser beams are useful attributes in certain types of application. Most laser beams are well-collimated with no more than milliradian divergence and most of the intensity is typically within a millimetre cross-section. The exact distribution of intensity within the beam is determined by the laser mode structure, and in the simplest situation consists of an essentially Gaussian distribution. The beam can generally be focused down to a diffraction limit where its diameter is of the same order as the wavelength.

This is especially significant for microsampling applications, where sample volumes can be of the order of $1 \mu \mathrm{l}$. Indeed in laser-based methods for the analysis of HPLC eluent, sample volumes of a few nanolitres are possible. In the microanalysis of heterogeneous solids such as minerals or corroded metals, the precision of the laser beam as probe is also significant where it is used for ablation of surface for analysis. ${ }^{2}$ Where highpower pulses are used to ablate successive layers of such materials, the analysis additionally provides depth profile data. The ability to deliver laser beams into optical fibres facilitates a number of biological applications such as remote fibre fluorimetry.

The best measure of the intensity experienced by a sample is the irradiance, defined as the power delivered per unit crosssectional area of the beam. It is principally the small dimensions of the beam which give rise to the very high irradiances delivered by laser sources. A good continuouswave $(\mathrm{CW})$ argon ion laser, for example, produces an irradiance of about $10^{7} \mathrm{~W} \mathrm{~m}^{-2}$. However, this can be increased by focusing to the diffraction limit; in this instance irradiances of $10^{13} \mathrm{~W} \mathrm{~m}^{-2}$ can be achieved, representing intensities $10^{10}$ times larger than that of typical sunlight on the Earth's surface.

In terms of the irradiances produced by pulsed lasers, the highest figures are obtained through use of mode-locking and allied techniques, characterized by ultra-short pulses measured on the picosecond $\left(10^{-12} \mathrm{~s}\right)$ or even femtosecond $\left(10^{-15} \mathrm{~s}\right)$ timescale. Intensities of the order of $10^{16} \mathrm{~W} \mathrm{~m}^{-2}$ are readily 
attainable from a focused mode-locked argon laser, for example, representing a light flux equal to that associated with the interiors of stars. Spectroscopically, such high intensities prove useful both for the identification of weak spectral features and the study of intrinsically weak processes.

It is the high degree of monochromaticity of laser light which carries the most obvious significance for spectroscopic applications. This is epitomized by the development of highresolution electronic spectroscopy based on dye lasers, from which resolutions of below $0.1 \mathrm{~cm}^{-1}$ are now routinely attainable. Such spectra frequently reveal a wealth of fine structure not manifest by traditional methods. With suitable instrumentation, ring dye lasers can in fact offer a spectral resolution of $10^{-5} \mathrm{~cm}^{-1}$.

The unique attributes of laser radiation have engendered a wide range of new spectroscopic techniques, many characterized by processes in which samples display optical non-linearity in their response. ${ }^{3}$ This often reflects the fact that photons are not interacting singly with sample molecules, as in conventional spectroscopy, but in pairs or higher order groupings. Such effects are only observable under the intense photon flux provided by powerful pulsed lasers. Following the discovery of these effects there is now significant implementation for analytical purposes, particularly in connection with laser mass spectrometry.

Another important aspect of laser development has been the progressive reduction in achievable pulse duration. Off-theshelf commercial systems now offer reproducible femtosecond pulses. There is an inevitable price to pay for the time resolution thus achieved, however, and that is a loss of spectral resolution in accordance with the inexorable operation of the quantum mechanical Uncertainty Principle. Nonetheless, such pulses are very significant for laser spectroscopy, not least because they facilitate the study of processes which occur on a comparable timescale. ${ }^{5}$

As far as genuine chemistry is concerned, most such processes directly involve interaction with light. The range of atomic motion over picosecond times is necessarily very limited, allowing only unimolecular processes such as electron transfer or bond fission to occur. The utility of laser studies is illustrated by the photodecomposition of ICN. Time-resolved measurement of laser-induced fluorescence following femtosecond excitation ${ }^{6}$ has shown that $\mathrm{I}-\mathrm{CN}$ bond fission occurs on a timescale of about $200 \mathrm{fs}$. Such information can only be obtained by using ultra-short pulsed laser instrumentation.

\section{References}

1 Walling, J. C. in Tunable Lasers, eds. Mollenauer, L. F., and White, J. C., Springer, Berlin, 1987, p. 331

2 Moenke-Blankenburg, L., Laser Microanalysis, Wiley, New York, 1989.

3 Levenson, M. D., and Kano, S. S., Introduction to Non-linear Laser Spectroscopy, Academic Press, London, 2nd edn., 1988.

4 Antonov, V. S., and Letokhov, V. S., in Laser Analytical Spectrochemistry, ed. Letokhov, V. S., Adam Hilger, Bristol, 1986.

5 Fleming, G. R., Chemical Applications of Ultrafast Spectroscopy, Clarendon Press, Oxford, 1986.

6 Rosker, M. K., Dantus, M., and Zewail, A. H., Science, 1988. 241, 1200 .

\title{
Current Aspects of Laser Instrumentation
}

\author{
Martin R. S. McCoustra \\ School of Chemical Sciences, University of East Anglia, Norwich NR4 7TJ
}

In discussing the technological aspects of laser spectroscopy, it would traditionally have been sufficient to consider only the laser light source. Today, the laser system must be considered as consisting both of the laser itself and of any associated diagnostics systems, as illustrated below.

\section{Tunable Coherent Light Sources in the Visible and Ultraviolet}

In recent years, there have been significant advances in the technology associated with the production of tunable coherent radiation in the visible and ultraviolet (UV). However, the organic molecular dye laser remains the mainstay of laser spectroscopy. With 25 years of development, dye lasers can be regarded as a mature technology and this is reflected in the increasing use of microprocessor control systems in commercial products. Fundamentally, however, in both continuouswave $(\mathrm{CW})$ and pulsed operation, the user is faced with the choice of one of two alternative cavity designs. In $\mathrm{CW}$ operation, either a standing wave or a ring cavity is used with birefringent filters as wavelength-selective elements. The latter offers narrower linewidth operation in a broadband cavity, but with a more complex cavity design. Both designs can offer single-mode, ultra-narrow linewidth $\left(0.00002 \mathrm{~cm}^{-1}\right)$ operation by the addition of further optical elements to the cavity. For pulsed operation, the choice is between a littrow or a grazing incidence cavity using a diffraction grating for wavelength selection. Linewidths are limited to the Fourier transform limit of the laser pulsewidth. Cavity design can have a substantial effect on the observed linewidth, a simple littrow dye laser offering a naturally broader output than a grazing incidence cavity, although this can be reduced by the addition of an intracavity etalon. Transform limited linewidth can be achieved by using alternative cavity designs. ${ }^{1,2}$ In both $\mathrm{CW}$ and pulsed operation, the output power is ultimately determined by the pump power. Conversion efficiencies of about $30 \%$ are expected for efficient laser dyes. The use of non-linear optical techniques such as second harmonic generation (SHG), sumfrequency generation (SFG) and third harmonic generation (THG) allows extension of the operating frequency ranges of both pulsed and CW dye lasers into the UV and vacuum ultraviolet (VUV) ${ }^{3,4}$ Naturally, the conversion efficiencies obtained from CW systems are lower than those from pulsed systems. . $^{3,4}$

While it is likely that the dye laser will remain the major source of tunable coherent radiation for the next few years, the challenge of solid-state sources is apparent. In particular, transition metal ion vibronic state lasers ${ }^{5}$ and the optical parametric oscillator (OPO) are seen as alternatives to the dye laser. In the former, the gain medium is a transition metal ion doped into an inert support matrix. Two systems have proved particularly attractive for operation in the visible: the alexandrite laser $\left(\mathrm{Cr}^{3+}\right.$ doped into $\mathrm{BeAl}_{2} \mathrm{O}_{4}, 700-800 \mathrm{~nm}$ operation) and the titanium : sapphire laser $\left(\mathrm{Ti}^{3+}\right.$ doped into $\mathrm{Al}_{2} \mathrm{O}_{3}, 670-$ $1100 \mathrm{~nm}$ operation). Many view the latter as the ideal replacement for the dye laser. When offered with frequency shifting (SHG, THG and SFG) options, a titanium:sapphire laser could be capable of covering the entire $200-1100 \mathrm{~nm}$ region, as illustrated in Fig. 1, with appreciable output powers. A similar tunability can be acheived with the alexandrite laser. 


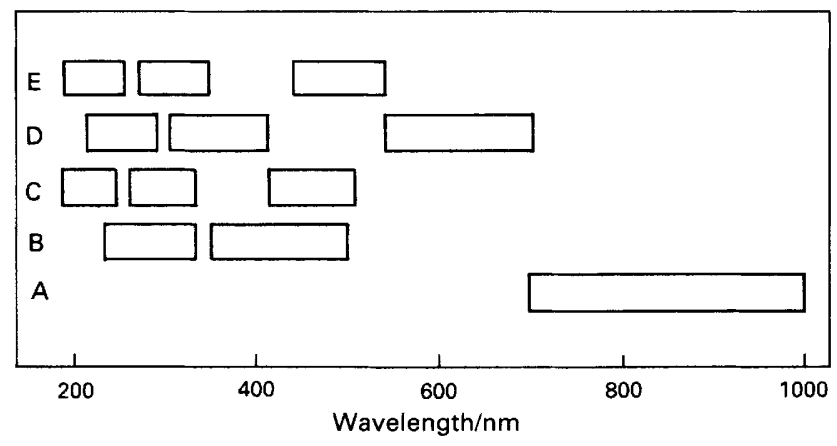

Fig. 1 Tuning ranges of a hypothetical Nd: YAG second harmonic pumped titanium:sapphire laser. A, Fundamental; B, second and third harmonics; $C$, sum frequency generation with $1064 \mathrm{~nm}$; D, first anti-Stokes Raman shifted in $\mathrm{H}_{2}$; and $\mathrm{E}$, second anti-Stokes Raman shifted in $\mathrm{H}_{2}$

Further, neither of these lasers exhibits the photochemical stability and toxicity problems of dye lasers.

In the OPO, coherent tunable radiation is generated by a process that can be considered the reverse of SFG; a single fixed input frequency is split into two tunable outputs. In the near-IR, OPO action has been exhibited in lithium iodate and lithium niobate, while in the visible, urea ${ }^{6}$ and $\beta$-barium borate $(\mathrm{BBO})^{7}$ show promise as UV pumped OPOs. Clearly, nonlinear frequency shifting techniques could be applied to OPOs to extend their operating range into the UV.

\section{Modern Diagnostics}

There are four parameters (laser wavelength, bandwidth, output power/energy and pulsewidth) of any operating laser system, a detailed knowledge of which can prove invaluable to the laser spectroscopist. Today, a wide variety of tools are available for measuring these parameters.

For the spectroscopist, wavelength and bandwidth measurement are of primary importance. The spectroscopic measurement of laser wavelengths in the visible and UV is relatively easy. Measurements of the absorption spectra of standard materials, such as iodine, nitrogen dioxide, nitrogen monoxide or carbon monoxide are especially useful in this respect. Alternatively, measurements can be made of the widely spaced, narrow atomic spectral features observed in the optogalvanic spectroscopy (OGS) of hollow cathode discharges in rare gases and metals such as iron and uranium. The latter especially offers the possibility of calibration of the laser over a wider spectral range than with rare gas discharges.

A number of alternative instrumental techniques are on offer, relying on either dispersive or interferometric methods. In the former, the radiation is dispersed using a prism or grating monochromator. The practical application of dispersive techniques is limited by the size of the devices necessary to achieve the desired resolution. Moreover, there are problems associated with optical damage to components and with wavelength inaccuracies introduced by incomplete illumination of the dispersive element by a narrow, collimated beam.

A number of interferometric approaches have been described. ${ }^{8-10}$ For CW lasers, any of these methods can be applied. With pulsed lasers, the choice is more limited. ${ }^{10}$ Michelson, Fabry-Perot and Fizeau interferometers all offer wavelength accuracies of typically 1 part in $10^{6}$, although 1 part in $10^{8}$ can be achieved by using a frequency-stabilized $\mathrm{He}-\mathrm{Ne}$ laser standard.

With a growing interest in ultra-high resolution spectroscopy and in Doppler free spectroscopic techniques, measurement of bandwidth is an obvious performance indicator for ultranarrow bandwidth $\mathrm{CW}$ lasers. Moreover, with the use of transform-limited bandwidth pulsed lasers increasing, correct operation can only be assessed by bandwidth measurements.
Although dispersive techniques can be employed with broadband sources, for narrowband and ultra-narrowband lasers the Fabry-Perot interferometer (FPI) is the device of choice. By using scanning FPIs with CW lasers, or a number of fixed FPIs with different free spectral ranges for pulsed lasers, the measurement of laser bandwidth becomes routine. With the latter type of device, simultaneous wavelength and bandwidth measurements are feasible.

While measurement of laser wavelength and bandwidth might be of primary spectroscopic importance, the routine measurement of power and/or pulse energy can prove a useful diagnostic, especially during the alignment of dye lasers. The choice of measurement device is determined largely by the range of power/energy to be measured and whether the source is pulsed or $\mathrm{CW}$. At high $\mathrm{CW}$ powers and high average pulsed powers $\left(>10^{-3} \mathrm{~W}\right)$, calorimetric detectors are the norm. Surface absorbing devices are preferred for rapid response, but are limited by a low damage threshold (about $10^{10} \mathrm{~W} \mathrm{~m}^{-2}$ ). At even higher powers, volume absorbing detectors, exhibiting much higher damage thresholds (about $10^{15} \mathrm{~W} \mathrm{~m}^{-2}$ ), are used.

At low powers, devices capable of both $\mathrm{CW}$ power and pulsed energy measurement are common. These are classified into pyroelectric and quantum detectors. The former employ lithium tantalate sensors which directly convert the heating effect of a laser pulse into an electrical signal. These are capable of energy measurement in the nanojoule to millijoule range, limits being determined by the low sensitivity and low damage threshold of the pyroelectric media. Continuous-wave powers can also be measured by appropriate modulation of the continuous output. As with calorimeters, their spectral response is flat in the visible and near-UV. For the measurement of picojoule or lower energies, the high sensitivity of quantum devices is necessary. Photomultiplier tube (PMT) and photodiode (PD) detectors offer the possibility of covering the entire range of pulse energies from a single photon to levels comparable to those of pyroelectric detectors. The PMTs and PDs do, however, exhibit variations in detection efficiency with wavelength. This effect is lessened and UV/VUV response of quantum devices improved by the use of a quantum converter.

With the growing availability of pyroelectric and PD arrays, there has been a recent increase in interest in the measurement of the distribution of power/energy over the profile of the laser beam. ${ }^{11.12}$ This makes it possible to determine whether the laser is operating on a single spatial mode or whether there are contributions to the power/energy distribution from higherorder modes. It can give the user a guide to that formerly elusive quantity, beam quality.

The measurement of laser pulsewidth is as fundamental to spectroscopic experiments in the time domain as the other parameters discussed, particularly with ultrafast laser systems. Measurements of pulsewidth and the shot-to-shot variation of such width can also prove an invaluable diagnostic on nanosecond laser systems.

For long pulse ( $>1 \mathrm{~ns}$ ) systems, a simple reverse biased PD detector and good oscilloscope are sufficient for pulsewidth measurement. Several low-cost PDs offer reasonable rise time and bandwidth characteristics, although avalanche photodiodes are preferred. For shorter pulses ( $<1 \mathrm{~ns})$, pulsewidth measurement relies on autocorrelation methods, employing either a scanning or a single shot device. ${ }^{13}$

\section{Conclusion}

It can be expected that in the next two or three years, the dye laser will see its dominance as the main source of tunable coherent radiation in the visible eroded by the growing use of solid-state devices. This is eagerly awaited by the laser spectroscopic community. It is also likely that in this period, an increased effort will be made in developing the laser diagnostic techniques to the extent of them becoming routine. 


\section{References}

1 Littman, M. G., Opt. Lett., 1978, 3, 138.

2 Ewart, P., and Meacher, D. R., Opt. Commun., 1989, 71, 197.

3 Wallenstein, R., in Frontiers of Laser Spectroscopy of Gases, eds. Alves, A. C. P., Brown, J. M., and Hollas, J. M., Kluwer Academic, Dordrecht, 1988, p. 53.

4 Stoicheff, B. P., in Frontiers of Laser Spectroscopy of Gases, eds. Alves, A. C. P., Brown, J. M., and Hollas, J. M., Kluwer Academic, Dordrecht, 1988, p. 63.

5 Messenger, H. W., Laser Focus World, 1990, 26(6), 69.
6 Catella, G. C., Bohn, J. H., and Luken, J. R., IEEE J. Ouantum Electron., 1988, 24, 1201

7 Ebrahimzadeh, M., Henderson, A. J., and Dunn, M. H., IEEE J. Quantum Electron., 1990, 26, 1241.

8 Cotnoir, L. J., Laser Focus World, 1989, 25(4), 109.

9 Snyder, J. J., and Hansch, T. W., Laser Focus World, 1990 , 26(2), 69

10 Reiser, C., Proc. SPIE, Int. Soc. Opt. Eng., 1988, 912, 214.

11 Fleischer, J., Laser Focus World, 1989, 25(4), 131.

12 Messenger, H. W., Laser Focus World, 1990, 26(10), 30.

13 Wyatt, R., and Marinero, E. E., Appl. Phys., 1981, 25, 297.

\title{
Laser Electronic Spectroscopy of Large Molecules
}

\author{
J. Michael Hollas \\ Department of Chemistry, University of Reading, Whiteknights, P.O. Box 224, Reading RG6 $2 A D$
}

Studies in molecular electronic spectroscopy can be divided into those of structure and those of dynamics. This paper is concerned entirely with structure.

The electronic transitions involved are all between the ground state $S_{0}$ and the first excited singlet state $S_{1}$. All the results discussed involve fluorescence from $S_{1}$ and a high quantum yield is desirable.

While the use of lasers has revolutionized spectroscopy in general, the incorporation of supersonic jets has revolutionized electronic spectroscopy of large molecules in particular. Unless otherwise indicated, all of the spectra discussed here have been obtained with the molecules injected into a supersonic jet of helium in which their rotational and vibrational temperatures are reduced to, typically, 10 and $100 \mathrm{~K}$, respectively. The resulting simplification of otherwise congested electronic spectra of large molecules is dramatic.

\section{Fluorescence Excitation}

\section{Experimental Techniques}

In fluorescence excitation (FE) the laser radiation is tuned through the absorption spectrum and the total, undispersed fluorescence monitored. The FE spectrum closely resembles the absorption spectrum and gives information on vibrational energy levels in $S_{1}$.

\section{Single Vibronic Level (or Dispersed) Fluorescence}

In single vibronic level fluorescence (SVLF) the laser is tuned to a particular absorption band, thereby populating a single vibronic level in $S_{1}$. Under the collision-free conditions in the supersonic jet, fluorescence occurs from that level only and is dispersed by a scanning spectrometer.

The spectra discussed here are mostly SVLF spectra and have been obtained with a Molectron nitrogen/dye, a Quantel ND:YAG/dye or a JK/Quanta-Ray Nd:YAG/dye laser system with a frequency doubling and/or mixing facility to produce near-ultraviolet laser radiation.

\section{General Methods of Structure Determination}

If we wish to obtain the potential energy curve in the ground electronic state of a diatomic molecule it is essential to determine as many vibrational energy levels as possible. Ideally some of these should be in the region close to dissociation. The most effective way of obtaining a large number of vibrational levels is by fluorescence from an excited electronic state and relying on the Franck-Condon principle to confer intensity on transitions to high-lying levels.

In polyatomic molecules, if we require structural infor- mation in the ground electronic state, exactly the same principle applies. We use fluorescence from an excited state to access as many ground state vibrational levels as possible and use these to construct a potential function.

There are three types of vibration in polyatomic molecules which are of particular structural importance. The first is inversion, as in aniline (I) (see Fig. 1). In the $\mathrm{S}_{0}$ state there is a

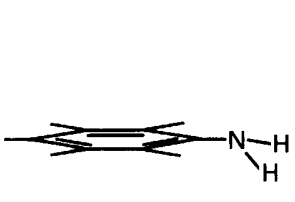

(I)

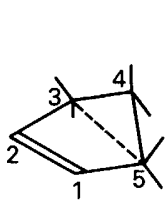

(II)

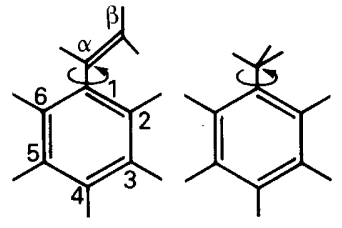

(III)
(IV)<smiles>Cc1c(C)c(F)c(C(C)(C)C)c(F)c1C</smiles>

(V)<smiles>CC(C)=C(C)c1c(C)c(C)c(C)c(F)c1C</smiles>

(VI)<smiles>CC(C)=C(C)C1=C(C)C(C)=C(C)C(C)=C(F)C1C</smiles>

(VII)<smiles>CC(C)=C(C)c1c(C)c(C)c(C)c(C(C)(C)C)c1C</smiles>

(VIII)<smiles>CC(C)=C(C)c1c(C)c(C)c(C)c(C)c1C(C)(C)C</smiles>

(IX)<smiles>Cc1c(C)c(C)c2c(c1C)C(C)(C)C(C)(C)C2(C)C</smiles>

(X)<smiles>Cc1c(C)c(C)c2c(c1C)O[B]O2</smiles>

(XI)<smiles>Cc1c(C)c(C)c2c(c1C)OC(C)O2</smiles>

(XII)<smiles>Cc1c(C)c(C)c2c(c1C)C(C)(C)OC2(C)C</smiles>

(XIII)<smiles></smiles>

(XIV)<smiles>CC1=C(C)C(C)=C2OCOC2=C(C)C(C)=C1C</smiles>

(XV)
Fig. 1 Structures of the compounds studied

pyramidal configuration about the nitrogen ${ }^{1}(c f$. ammonia, but the pyramid is flatter). The potential function for the $\mathrm{NH}_{2}$ inversion vibration shows two equivalent minima, corresponding to the hydrogen atoms being 'up' or 'down', and is Wshaped. The form of the potential function often used for an inversion vibration is 


$$
V(x)=A x^{2}+B x^{4}
$$

with $A$ negative and $B$ positive. The height of the energy barrier to planarity is $A^{2} / 4 B$.

The second type is a ring-puckering vibration, as in cyclopentene (II) (Fig. 1) in $S_{0}$. The carbon atom in the 4 position is not coplanar with the others. As for inversion, there are two configurations, with C(4) 'up' or 'down', having identical energy. The potential for the ring-puckering vibration, which takes $\mathrm{C}(4)$ from the 'up' to the 'down' position, is again W-shaped and the form assumed for it is that in eqn. (1).

The third type of vibration involves a torsional, or internal rotational, motion of one part of the molecule relative to another. Examples are the motion of the vinyl group relative to the benzene ring in styrene (III) and of the methyl group in toluene (IV) and 2-fluorotoluene (V) (Fig. 1). All torsional potentials must be repetitive for each change of torsional angle $\phi$ by $2 \pi$ and the general form is usually assumed to be

$$
V(\phi)=\frac{1}{2} \sum_{n} V_{n}(1-\cos n \phi)
$$

where $n$ is an integer. Which term is dominant depends on how often $V(\phi)$ repeats during a complete rotation of the group. In styrene, toluene and 2-fluorotoluene, $V_{2}, V_{6}$ and $V_{3}$, respectively, are dominant.

If the energy barrier is sufficiently low in either a W-shaped or torsional potential, quantum mechanical tunnelling through the barrier can occur, producing splittings and irregularities in the vibrational levels.

\section{Results}

\section{Styrene}

The SVLF spectrum of styrene, ${ }^{2,3}$ with the laser tuned to the $0_{0}^{0}$ band (pure electronic transition), shows transitions to vibrational levels in the $C(1)-C(\alpha)$ torsional vibration in $S_{0}$, with even values of the vibrational quantum number $v$ up to 10: the vibrational selection rule restricts $\Delta v$ to even values only. Levels with odd values of $v$ can be accessed through fluorescence from a different vibrational level in $S_{1}$. Fitting the levels to the potential in eqn. (2), with $V_{2}$ and $V_{4}$ terms only, gives

$$
V(\phi) / \mathrm{cm}^{-1}=\frac{1}{2}[1070(1-\cos 2 \phi)-275(1-\cos 4 \phi)]
$$

The large, negative value of $V_{4}$ results in a very flat-bottomed potential. While conjugation favours planarity, steric hindrance does not and the result of these two competing effects is that the molecule is planar, but only just: it requires relatively little energy to twist the vinyl group out-of-plane. The torsional barrier height is $1070 \mathrm{~cm}^{-1}$ (or $12.8 \mathrm{~kJ} \mathrm{~mol}^{-1}$ ).

\section{3-Fluorostyrene}

This molecule can exist as the cis (VI) or trans (VII) rotamer (Fig. 1). Both have been discovered ${ }^{4}$ and SVLF spectra with excitation in both of their $0_{0}^{0}$ bands obtained. Eight torsional levels of the cis and two of the trans rotamer were fitted to a potential containing $V_{1}, V_{2}$ and $V_{4}$ terms

$$
\begin{aligned}
V(\phi) / \mathrm{cm}^{-1}=\frac{1}{2}[220(1-\cos \phi)+ & 1040(1-\cos 2 \phi) \\
& -247(1-\cos 4 \phi)]
\end{aligned}
$$

The value of $V_{1}$ represents the energy difference between the cis and trans rotamers, there being a 3:1 cis: trans ratio at room temperature. As for styrene, the large, negative value of $V_{4}$ results in a very flat-bottomed potential: both rotamers are planar, but only just.

\section{3-Methylstyrene}

The cis (VIII) (Fig. 1) and trans rotamers of 3-methylstyrene are present in approximately equal amounts. ${ }^{3.5}$ Only the methyl torsional potential will be discussed here.

In the SVLF spectrum of the cis rotamer many torsional
Table 1 Methyl torsional potentials in the $S_{0}$ and $S_{1}$ electronic states of methylstyrenes compared with toluene
$\mathrm{S}_{0}$

cis-3-Methylstyrene trans-3-Methylstyrene 4-Methylstyrene Toluene

\begin{tabular}{ccccc}
\cline { 1 - 1 }$/ \mathrm{cm}^{-1}$ & $V_{6} / \mathrm{cm}^{-1}$ & & $V_{3} / \mathrm{cm}^{-1}$ & $V_{6} / \mathrm{cm}^{-1}$ \\
45.0 & 0 & & 165 & -13 \\
52 & 19 & & 79 & 5 \\
- & -17.0 & & - & -17.0 \\
- & -5.0 & - & -25.0 \\
\hline
\end{tabular}

levels in $\mathrm{S}_{0}$ have been obtained, ${ }^{3}$ resulting in the values of $V_{3}$ and $V_{6}$ in Table 1 . Fewer levels have been observed for the trans rotamer but values of $V_{3}$ and $V_{6}$ have also been determined. ${ }^{3}$ The value of $V_{3}$ represents the torsional energy barrier. This has been shown, ${ }^{3,5}$ from FE spectra of 3methylstyrene, to increase considerably in $S_{1}$. Table 1 also gives the energy barrier $\left(=\left|V_{6}\right|\right)$ in $\mathrm{S}_{0}$ and $\mathrm{S}_{1}$ of 4 methylstyrene $^{6}$ and toluene. ${ }^{7,8}$ Comparisons show that 4 substitution in toluene makes little difference to the low barrier in $S_{0}$ and $S_{1}$ but that 3-substitution increases the barrier substantially in $S_{0}$ and even more so in $S_{1}$.

\section{2-Methylstyrene}

2-Methylstyrene is assumed to exist predominantly as the trans rotamer (IX) (Fig. 1), as is the case for 2-fluorostyrene. The SVLF spectrum, with excitation in the $0_{0}^{0}$ band, ${ }^{3}$ shows no activity of the methyl torsional vibration but there is a long progression in the vinyl $\mathrm{C}(1)-\mathrm{C}(\alpha)$ torsional vibration with $\Delta v$ even and an intensity maximum at $v=4$. Vinyl torsional levels with $v$ odd have been observed in other SVLF spectra and all levels fitted to the potential function

$$
V(\phi) / \mathrm{cm}^{-1}=\frac{1}{2}[670(1-\cos 2 \phi)-420(1-\cos 4 \phi)]
$$

It can be shown that, if $V_{2}<4\left|V_{4}\right|$, there is a barrier to planarity. This is the case for trans-2-methylstyrene. The barrier height is $152 \mathrm{~cm}^{-1}$ and the vinyl group is about $33^{\circ}$ out of plane in $\mathrm{S}_{0}$. This considerable non-planarity contrasts with trans-2-fluorostyrene ${ }^{9}$ which is quasi-planar: there is a barrier but it is only $15 \mathrm{~cm}^{-1}$, just below the zero-point level.

Indan, 1,3,2-Benzodioxaborole, 1,3-Benzodioxole and Phthalan In indan (X), 1,3,2-benzodioxaborole (XI), 1,3-benzodioxole (XII) and phthalan (XIII) (Fig. 1) the structural interest relates to whether the skeletal atoms of the partly saturated fivemembered ring are coplanar with the benzene ring. The vibrations of the greatest structural importance turn out to be the puckering vibration of the group in position 2 , in indan and 1,3,2-benzodioxaborole, and the twisting vibration of the atoms or groups in positions 1 and 3, in 1,3-benzodioxole and phthalan.

The SVLF spectrum of indan, with excitation in the $0_{0}^{0}$ band, shows an evenly spaced progression in the puckering vibration of the $\mathrm{CH}_{2}$ group in position 2 , with $v$ even and odd. ${ }^{10}$ This selection rule shows that the molecule is strongly puckered in $\mathrm{S}_{0}$. The barrier to planarity is so high $\left(1980 \mathrm{~cm}^{-1}\right)$ that there is no observed tunnelling through it up to $v=4$. The nonplanarity is due to the resistance of the adjacent $\mathrm{CH}_{2}$ groups to being eclipsed. Non-planarity is preserved in $\mathrm{S}_{1}$ but there is a lower barrier resulting in tunnelling splitting being observed.

With excitation in the $0_{0}^{0}$ band of $1,3,2$-benzodioxaborole the SVLF spectrum shows an evenly spaced progression in the $\mathrm{BH}-$ puckering vibration with $v$ even, only, showing that the molecule is rigidly planar. ${ }^{11}$ The planarity is due to the delocalization of the $\pi$-electrons on the carbon and oxygen atoms of the five-membered ring into the otherwise empty boron $2 p$ orbital. The molecule is planar in $S_{1}$ also.

The SVLF spectra of 1,3-benzodioxole ${ }^{12}$ and phthalan, ${ }^{13}$ with $0_{0}^{0}$ band excitation, are more complex. They both show a prominent band at 102 and $92 \mathrm{~cm}^{-1}$, respectively, to low 
wavenumber of the $0_{0}^{0}$ band and in neither instance is the interval repeated. These bands are assigned to two quanta of an anharmonic twisting vibration of the oxygen atoms, in 1,3benzodioxole, or the carbon atoms in phthalan, in the 1 and 3 positions. Several levels of this vibration in each molecule have been fitted to the $W$-shaped potential function of eqn. (1). Both molecules are twisted: the barrier to planarity of the skeleton is $157 \mathrm{~cm}^{-1}$ in 1,3 -benzodioxole and $137 \mathrm{~cm}^{-1}$ in phthalan.

\section{Tropolone}

Tropolone (XIV) (Fig.1) presents an interesting example of internal hydrogen bonding. Is the hydrogen atom involved more closely attached to one oxygen atom than the other, as in XIV, or midway between them, as in XV? If XIV is the more stable structure, the hydrogen atom might be attached more strongly to either oxygen atom and motion of the hydrogen from one oxygen atom to the other results in a $W$-shaped potential with structure $\mathbf{X V}$ corresponding to the top of the energy barrier.

In the $S_{1}-S_{0}$ gas phase absorption spectrum, pairs of bands occur throughout. The separation within each pair is about $19 \mathrm{~cm}^{-1}$ and was interpreted ${ }^{14}$ as involving $v_{\mathrm{H}}^{\prime}=0-v_{\mathrm{H}}^{\prime \prime}=0$ and $v_{\mathbf{H}}^{\prime}=1-v_{\mathbf{H}}^{\prime}=1$ transitions between levels of the hydrogen-bonding vibration $v_{\mathrm{H}}$. The splitting, reduced to $2 \mathrm{~cm}^{-1}$ in tropolone-OD,${ }^{14}$ was interpreted in terms of a $W$ shaped potential in $S_{0}$ and $S_{1}$ with tunnelling through the barrier to produce a $v_{\mathrm{H}}=1-0$ splitting, which is $19 \mathrm{~cm}^{-1}$ $\left(2 \mathrm{~cm}^{-1}\right.$ in tropolone-OD) greater in $\mathrm{S}_{1}$ than in $\mathrm{S}_{0}$. This interpretation is supported by laser-induced FE and SVLF spectra in a supersonic jet ${ }^{15}$ and in the collision-free gas phase. $^{16}$

It has been estimated ${ }^{17}$ that the height of the barrier between the more stable structure XIV and the symmetrical structure
$\mathrm{XV}$ is $4790 \mathrm{~cm}^{-1}$ in $S_{0}$ and that the $v_{\mathbf{H}}^{\prime \prime}=1-0$ splitting is $\leqslant 0.2 \mathrm{~cm}^{-1}$

\section{References}

1 Hollas, J. M., Howson, M. R., Ridley, T., and Halonen, L., Chem. Phys. Lett., 1983, 98, 611.

2 Hollas, J. M., Musa, H., Ridley, T., Turner, P. H., Wiesenberger, K. H., and Fawcett, V., J. Mol. Spectrosc., 1982, 94, 437.

3 Hollas, J. M., and Taday, P. F., J. Chem. Soc., Faraday Trans., in the press.

4 Hollas, J. M., and bin Hussein, M. Z., Chem. Phys. Lett., 1989 , 154, 228.

5 Grassian, V. H., Bernstein, E. R., Secor, H. V., and Seeman, J. I., J. Phys. Chem., 1989, 93, 3470.

6 Hollas, J. M., and Taday, P. F., J. Chem. Soc., Faraday Trans., 1990, 86, 217.

7 Rudolph, H., Dreizler, H., Jaeschke, A., and Wendling, P., Z. Naturforsch. A, Astrophys. Phys. Phys. Chem., 1967, 22, 940 .

8 Breen, P. J., Warren, J. A., Bernstein, E. R., and Seeman, J. I., J. Chem. Phys., 1987, 87, 1917.

9 Hollas, J. M., and bin Hussein, M. Z., Chem. Phys. Lett., 1989, 154, 14.

10 Hassan, K. H., and Hollas, J. M., J. Mol. Spectrosc, , 1991, 147, 100.

11 Alves, A. C. P., Hassan, K. H., and Hollas, J. M., J. Chem. Soc., Faraday Trans., 1990, 86, 3341.

12 Hassan, K. H., and Hollas, J. M., Chem. Phys. Lett., 1989, 157, 183.

13 Hassan, K. H., and Hollas, J. M., Chem. Phys. Lett., 1990, 169. 179.

14 Alves, A. C. P., and Hollas, J. M., Mol. Phys., 1972, 23, 927.

15 Tomioka, Y.. Ito, M., and Mikami, N., J. Phys. Chem., 1983. 87, 4401 .

16 Alves, A. C. P.. Hollas, J. M., Musa, H., and Ridley, T., J. Mol. Spectrosc., 1985, 109, 99.

17 Redington, R. L., J. Chem. Phys., 1990, 92, 6447.

\title{
Laser Mass Spectroscopy
}

\author{
Kenneth W. D. Ledingham \\ Department of Physics and Astronomy, University of Glasgow, Glasgow G12 800
}

Laser mass spectroscopy is an ultra-sensitive analytical technique which uses one or more lasers in conjunction with mass spectrometers of normally time-of-flight type, although quadrupole or magnetic sector types are also used. The lasers are utilized to produce ions from a solid source or are used to postionize neutral atoms or molecules, themselves produced by a second laser or an ion beam. There are a number of different arrangements of lasers and mass spectrometers and in this paper three principal configurations will be identified and described: laser microprobe analysis; laser post-ionization of neutrals either resonant or non-resonant; and a new technique which has been called resonant laser ablation.

\section{Laser Microprobe Analysis}

This type of laser mass spectroscopy is alternatively called LIMA (Kratos) ${ }^{1}$ or LAMMA (Leybold). ${ }^{2}$ Usually the sample is in a solid form and is gasified (desorbed or ablated) by a laser in vacuum, normally a quadrupled Nd: YAG laser operated at $266 \mathrm{~nm}$. A large number of positive or negative ions are created in addition to greater numbers of neutral atoms or molecules. The ions alone are analysed using a time-of-flight (TOF) mass spectrometer of linear or reflectron type. Typically the mass resolution of a linear TOF spectrometer is about 100 , whereas the reflectron TOF spectrometer can have a resolution as high as 3000 . This type of laser mass spectroscopy is particularly suited for the detection of molecules because the laser desorption can be made soft, giving a large unfragmented parent peak. LIMA and LAMMA give analysis commonly at ppm levels although matrix problems abound. If non-quantitative, multi-element analysis is required the laser microprobe is an ideal instrument.

\section{Laser Post-ionization of Neutrals}

It was stated above that in the desorption or ablation process many more neutrals are created than ions (by several orders of magnitude). ${ }^{3}$ These neutrals can then be ionized nonresonantly using a quadrupled Nd: YAG or an excimer laser resulting in a process called SALI (surface analysis by laser ionization $)^{4}$ with increased sensitivity for multi-element analysis. If the neutrals are ionized resonantly the process is called resonant ionization mass spectroscopy (RIMS). This technique was developed in the 1970 s principally by Hurst and Payne at Oak Ridge ${ }^{5}$ and by Letokhov and co-workers in Moscow. ${ }^{6}$ Although the samples to be analysed can be in solid, liquid or gaseous state, the preferred form is solid and both stable and radioactive material can be assayed. If the sample is a solid then analysis using a modern RIMS arrangement consists of three steps. (a) The sample is gasified by either ion sputtering or laser desorption to form a plume of principally neutral atoms or molecules. $(b)$ The neutral atoms or molecules in the plume 
are resonantly ionized using one or more dye lasers, normally pulsed and broadband. For atoms this step is elementally very selective with isotopic selectivity being carried out in step $(c)$. Isobaric interferences which plague conventional mass spectroscopy are largely eliminated at this stage. (c) Finally, the ions created are mass analysed, normally using a TOF mass spectrometer which complements ideally the pulsed aspect of the resonant ionization step.

The particular advantages of RIMS over secondary ionization mass spectroscopy (SIMS) or other mass spectroscopic techniques are: $(a)$ elimination of isobaric interferences because the lasers are tuned to a specific element; $(b)$ greater sensitivity by several orders of magnitude because the neutrals are ionized often at saturation levels; and $(c)$ the analysis is essentially matrix free and hence RIMS is potentially the best technique for ultra-sensitive quantitative analysis.

The Glasgow RIMS instrument is shown schematically in Fig. $1{ }^{7}$ The TOF mass spectrometer is of reflectron type with a

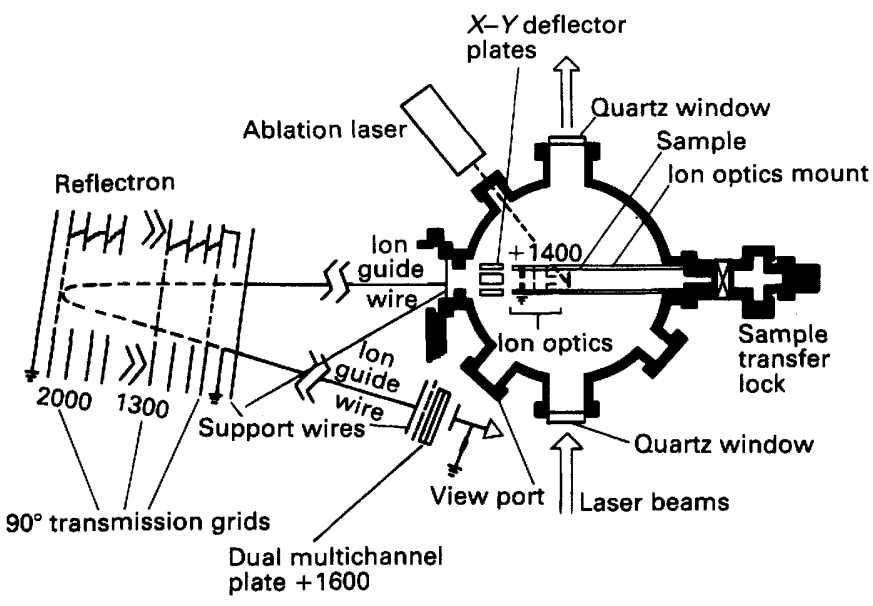

Fig. 1 Schematic diagram of the Glasgow RIMS instrument with reflectron TOF mass spectrometer. The ion guide wire increases the transmission

mass resolution of about 1000 for ions of about $40 \mathrm{u}$. For complete elemental coverage an $\mathrm{Nd}$ : YAG laser powering two dye lasers, one of which is frequency doubled, is required.

\section{Bulk Analysis by RIMS}

Typically the sensitivity of bulk analysis by RIMS is sub-ppm and does not show the large matrix problems encountered by SIMS, LIMA or LAMMA. Sub-ppb sensitivity down to parts in $10^{12}$ has been reported in the literature ${ }^{8}$ for stable isotopes. For radioactive isotopes in environmental monitoring, sample sizes as small as $10^{8}$ atoms of $\mathrm{Pu}$ have been detected ${ }^{9}$ and potentially this technique can detect down to $10^{6}$ atoms using high rep. rate $\mathrm{Cu}$ vapour lasers to pump the dye lasers.

\section{Profiling by RIMS}

Profiling is very important to the semiconductor industry where the concentration of particular atoms as a function of depth below the surface is measured. Of particular importance at present is the profiling of quantum well structures in $\mathrm{III} / \mathrm{v}$ semiconductors, particularly AlGaAs. In this instance depth resolution is the important parameter and already depth resolutions of about $45 \AA$ have been obtained. ${ }^{10}$

Secondary ionization mass spectroscopy is by far the most popular technique for profiling layered structures, but recently difficulties have become apparent because of the matrix problems associated with sputtering certain types of atoms in the presence of others. It is likely that RIMS will become important in the solution of these types of problems and also to detect impurities within the quantum wells. Profiling is normally carried out using argon or oxygen ion beams to etch away the layers followed by resonant lasers to ionize the sputtered neutrals. ${ }^{11}$

\section{Detection of Molecules by RIMS or SALI}

Resonant ionization mass spectroscopy is now well established as a means of detecting very low level atomic concentrations. Detection of low molecular concentrations is much more difficult because molecular spectroscopy is much more complicated than atomic spectroscopy. This problem can be alleviated to a large extent by cooling the molecules to about $10 \mathrm{~K}$ using a pulsed supersonic gas jet. Under these conditions, complex molecular spectra can be reduced to a few sharp atomic-like lines. The normal arrangement is desorption of the sample by a $\mathrm{CO}_{2}$ laser. The emitted neutral molecules suffer collisions with a supersonic beam of argon atoms the origin of which lies very close to the surface of the sample. The cold molecules are transported downstream where they are ionized by resonant or non-resonant lasers and hence detected by reflectron type mass spectrometers. The effectiveness of this technique has been demonstrated for large labile biomolecules, e.g., chlorophylls, porphyrins and peptides.

The apparatus for recording jet spectra by laser desorbing into a jet followed by laser ionization is shown in Fig. $2 .^{12}$

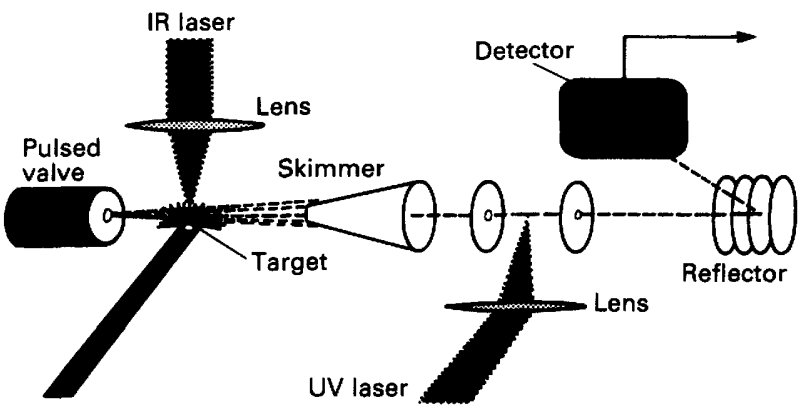

Fig. 2 Sample is irradiated by an IR laser to produce molecules which are seeded into a supersonic jet. These are post-ionized by a UV laser and then analysed by a reflectron TOF mass spectrometer

\section{Resonant Laser Ablation}

Resonant ionization mass spectroscopy is a complicated experimental configuration requiring at least two laser systems. It has been felt that a simpler arrangement which might come close to the sensitivity of RIMS would be resonant laser ablation (RLA). ${ }^{13}$ In this procedure a single tunable laser both ablates and resonantly ionizes the sample if the laser wavelength is chosen to match known resonant atomic transitions. In the RLA procedure adopted by McLean et al.,${ }^{13}$ a dye laser beam was moderately focused to give a beam spot of about $1 \mathrm{~mm}$ diameter and was directed at grazing incidence to the target, a semiconductor layer of $\mathrm{Al}_{x} \mathrm{Ga}_{1-x} \mathrm{As}$. As the laser wavelength was varied through known Al transitions, signal enhancements of greater than two orders of magnitude were observed. Recent work by Wang et al. ${ }^{14}$ has shown that the high ionization efficiency is due in part to collisional ionization in the plume.

\section{Conclusions}

Laser mass spectroscopy is an ultra-sensitive analytical technique with enormous potential. One of the problems associated with the technique which has been identified by instrument manufacturers is the use of dye lasers with their inherent bulkiness and operating difficulties. With the development of solid-state tunable lasers (Ti sapphire or optical parametric oscillators ${ }^{15}$ ) to replace dye lasers this difficulty can be overcome. 


\section{References}

1 Southon, M. J., Witt, M. C., Wallach, E. R., and Myatt, J., Vacuum, 1984, 34, 903.

2 Krier, G., Verdun, F. R., and Muller, J. F., Fresenius Z. Anal. Chem., 1985, 322, 379.

3 Fürstenau, N., Fresenius Z. Anal. Chem., 1981, 308, 201.

4 Becker, C. H., and Gillen, K. T., J. Opt. Soc. Am. B, Opt. Phys., 1985, 2, 1438.

5 Hurst, G. S., and Payne, M. G., Spectrochim. Acta, Part B, $1988,43,715$

6 Letokhov, V. S., Laser Analytical Spectrochemistry, Adam Hilger, Bristol, 1986.

7 Towrie, M., Drysdale, S. L. T., Jennings, R., Land, A. P. Ledingham, K. W. D., McCombes, P. T., Singhal, R. P., Smyth, M. H. C., and McLean, C. J., Int. J. Mass Spectrom. Ion Processes, 1990, 26, 309.

8 Bekov, G. I., Letokhov, V. S., and Radaev, V. N., J. Opt. Soc. Am. B, Opt. Phys., 1985, 2, 1554.
9 Krönert, U., Bonn, J., Kluge, H.-J., Ruster, W., Wallmeroth K., Peuser, P., and Trautman, N., Appl. Phys. B, 1985, 38, 65 .

10 Parks, J. E., Spaar, M. T., Beekman, D. W., Moore, L. J., and Cressman, P. J., RIS 88, IoP Conf. Ser. No. 94, Institute of Physics, Bristol, p. 197.

11 Arlinghaus, H. F., Spaar, M. T., and Thonnard, N., J. Vac. Sci. Technol. A, 1990, 8, 2318.

12 Grotemeyer, J., and Schlag, E. W., Acc. Chem. Res., 1989, 22 399.

13 McLean, C. J., Marsh, J. H., Land, A. P., Clark, A., Jennings, R., Ledingham, K. W. D., McCombes, P. T., Marshall, A., Singhal, R. P., and Towrie, M., Int. J. Mass Spectrom. Ion Processes, 1990, 96, R1.

14 Wang, L., Borthwick, I. S., Jennings, R., McCombes, P. T., Ledingham, K. W. D., Singhal, R. P., and McLean, C. J., RIS 90, IoP Conf. Ser. No. 114, Institute of Physics, Bristol, 1991, p. 455 .

15 Ledingham, K. W. D., and Singhal, R. P., J. Anal. At. Spectrom., 1991, 6, 73.

\section{Laser-induced Fluorescence}

\section{J. Pfab Chemistry Department, Heriot-Watt University, Edinburgh EH14 4AS}

Laser-induced fluorescence (LIF) spectroscopy was introduced about 20 years ago and has become a standard technique for recording the electronic spectra of atoms, diatomics, radicals and other polyatomic molecules in the gas phase and in lowtemperature crystalline or amorphous matrices. The detection of fluorescence against a zero background is intrinsically much more sensitive and easier than a measurement of absorbed light from small differences between incident and transmitted light intensities. ${ }^{1}$

The principle of the technique is based on the electronic excitation of a sample to a bound upper state that emits fluorescence with a finite quantum yield. Low fluorescence yields or small absorption cross-sections in the relevant spectral region can be overcome by using pulsed laser sources and focused beams capable of delivering high photon fluxes into the observation volume. In the gas phase at low pressure, where individual vibration-rotation states will generally be excited by a narrow bandwidth tunable laser, the fluorescence emitted will be resonance fluorescence, i.e., only the particular vibronic state tuned into resonance will emit downwards. The intensity and frequency distribution of the emitted light mirrors the ground state levels and their Franck-Condon overlap with the emitting level and also the spectroscopic selection rules.

At higher pressure, collisional relaxation during the lifetime of the excited state will lead to transfer of the excited state population to neighbouring levels which will then also contribute to the total fluorescence. With certain collision partners, where electronic energy transfer is facile, electronic quenching leads to a shortening of the observed fluorescence decay time and concomitant loss of the fluorescence intensity. In the condensed phase, emission occurs from a vibrationally relaxed upper electronic state.

The sensitivity of LIF in the gas phase is impressive and can be as high as $10^{6}$ molecules per cubic centimetre and quantum state. The detection limits achievable here depend on the number of quantum states occupied and will clearly be lowest for atomic and light diatomic species. Femto- to attomole sensitivity can be achieved in the condensed phase for highly fluorescent molecules. With pulsed excitation, single-photon LIF signals do not necessarily increase linearly with excitation intensity and the effects of optical saturation and power broadening are seen frequently. One of the most attractive features of the LIF technique is its relative technical simplicity. The most critical experimental requirement is efficient rejec- tion of scattered laser light. In two-photon LIF, the emitted light has approximately half the wavelength of the exciting radiation, rendering the discrimination of fluorescence and scattered laser light very easy. Brewster-angled entrance and exit windows, elaborate baffles and light skimmers and optical filters serve to minimize scattered laser light. Mirrors and lenses collect the luminescence and transfer the light to the sensitive area of the detector, usually a photomultiplier or multichannel detector such as a charge-coupled device (CCD) camera if very low light levels are to be detected. With pulsed excitation, standard gated boxcar averaging or transient recording techniques are needed, whereas continuous-wave (CW) excitation requires choppers and lock-in amplifiers to record the signal in analogue form. Pulsed tunable lasers offer advantages as excitation sources in LIF because the temporal shape of the fluorescence decay provides an additional useful source of information.

In the standard LIF experiment the emission is not spectrally dispersed. By scanning the tunable laser while monitoring the undispersed fluorescence as a function of excitation wavelength, fluorescence excitation spectra are recorded. Provided the fluorescence quantum yield remains constant, excitation spectra will be identical with absorption spectra, but emission yields frequently decrease towards shorter wavelengths as a consequence of increasing competition from non-radiative processes.

The first applications of LIF more than 20 years ago were in atomic and molecular spectroscopy. Among the diatomics, more precise molecular constants were obtained, for example with the halogens and interhalogens, and many new electronic states of unstable species such as NS or PO have been characterized. The discovery of new electronic systems or even the measurement of LIF spectra of radicals identified previously in discharges or during flash photolysis quickly had a major impact and led to a renaissance of electronic spectroscopy. Examples are: atoms such as $\mathrm{H}, \mathrm{O}, \mathrm{S}, \mathrm{Cl}, \mathrm{Br}$ and $\mathrm{I}$; diatomics such as $\mathrm{H}_{2}, \mathrm{OH}, \mathrm{SH}, \mathrm{CN}, \mathrm{CO}, \mathrm{NO}, \mathrm{SiH}, \mathrm{Be}_{2}$ and $\mathrm{V}_{2}$; triatomics such as $\mathrm{CH}_{2}, \mathrm{NH}_{2}, \mathrm{CF}_{2}, \mathrm{NO}_{2}, \mathrm{BO}_{2}$ and $\mathrm{SiH}_{2}$; and polyatomic radicals, for example $\mathrm{CH}_{3} \mathrm{O}, \mathrm{CH}_{3} \mathrm{~S}, \mathrm{CH}_{3} \mathrm{~N}$ $\mathrm{CH}_{2} \mathrm{CHO}$ and $\mathrm{C}_{6} \mathrm{H}_{5} \mathrm{CH}_{2}$. Small unstable species such as $\mathrm{HNO}$, $\mathrm{H}_{2} \mathrm{CS}$ and NCNO have been studied, and there is a vast range of larger molecules that includes transition metal compounds such as $\mathrm{CrO}_{2} \mathrm{Cl}_{2}$ and large, biologically relevant, molecules such as porphyrin and peptides that have been studied by LIF. 
The huge potential of marrying LIF spectroscopy with the supersonic jet-cooling technique became apparent around 1978 through the work of Levy. ${ }^{2}$ Cooling molecules to less than $5 \mathrm{~K}$ by free jet expansion with a rare gas simplifies dramatically the electronic spectra of polyatomic molecules by removing the spectral congestion characteristic of room temperature spectra. This approach is particularly useful in providing new information on the electronic spectra of radicals and unstable, weakly bound complexes. ${ }^{3}$ Laser-induced fluorescence is one of a number of laser spectroscopic techniques that permit structural and dynamic studies of van der Waals complexes and hydrogen-bonded adducts.

Laser-induced fluorescence as a diagnostic technique is currently playing a leading role in reaction and photodissociation dynamics. The major objective here is to probe directly the time evolution on a nano- or picosecond scale or the internal energy distribution of nascent products of a chemical reaction or photodissociation event. Where these products are diatomic fluorescent species such as $\mathrm{OH}$, NO or $\mathrm{CO}$, detailed information on rotational, vibrational, electronic and fine-structure populations can be obtained by LIF. By using polarized photolysis for alignment of parent molecules and polarized beams for LIF probing, vectorial and also scalar information can be deduced about the alignment and recoil direction of fragments. The study of Doppler-profiles of individual LIF transitions has recently permitted very detailed information to be obtained on the stereochemistry of dissociation processes.

The LIF technique has also been applied to probe the scattering and desorption of diatomic species from welldefined surfaces. ${ }^{4}$ One of the best studied systems is the interaction of NO with metal surfaces such as Pt, a system that is relevant in the catalysed oxidation of $\mathrm{NH}_{3}$ to $\mathrm{NO}$ or in the reduction of $\mathrm{NO}_{x}$ gases in the catalytic converter. At low surface temperature the NO has been shown by LIF and resonance enhanced multiphoton ionization to interact with the Pt surface by a trapping/desorption mechanism. ${ }^{4}$ Perhaps the most impressive examples of the use of LIF are in the optical probing of minority species such as $\mathrm{OH}, \mathrm{SiH}$ and $\mathrm{NO}$ in hostile environments, particularly in flames, discharges and plasmas. Here, concentration profiles can be established by exploiting the non-intrusive nature of the LIF probe and its high spatial resolution. T-profiles can be obtained from the Doppler-widths of atomic LIF lines. Numerous applications in combustion studies and more recently in work on plasma discharges of relevance in the semiconductor industry have shown the dramatic advances that this technique permits in high technology research.

Applications of LIF to the trace analysis of metals and air pollutants are also promising. The detection limit for lead in aqueous solution using atomic LIF is near to $30 \mathrm{fg} \mathrm{ml}^{-1}$ and NO in air can be analysed at the parts per trillion level. Laserinduced fluorescence also appears to be capable of improving detection limits in liquid chromatography where the ability to monitor sub-microlitre volumes with focused beams offers advantages. Remote sensing of oil pollution or algal blooms from high-flying aircraft using the fluorescence LIDAR technique (light detection and ranging) or the probing of alkali and alkaline earth metal atoms in the upper atmosphere by examining altitude resolved back-scattered fluorescence are two particularly interesting applications of LIF.

\section{References}

1 Zare, R. N., Science, 1984, 226, 298.

2 Levy, D. H., Sci. Am., 1984, February, 68.

3 Miller, T. A., Science, 1984, 223, 545.

4 Lin, M. C., and Ertl, G., Annu. Rev. Phys. Chem., 1986, 37, 587.

\title{
Multiphoton Absorption Spectroscopy
}

\author{
Michael N. R. Ashfold \\ School of Chemistry, University of Bristol, Bristol BS8 1TS
}

\section{Resonance Enhanced Multiphoton Ionization Spectroscopy}

The development of reliable, high powered, pulsed, tunable dye lasers has opened the way to a range of multiphoton excitation spectroscopic techniques. ${ }^{1}$ Of these, the most widely used to date has been resonance enhanced multiphoton ionization (REMPI) spectroscopy. ${ }^{2,3}$ The principles, and application, of this technique are illustrated in Fig. 1. Visible (or near-ultraviolet) radiation of frequency $v$ is focused into a low pressure sample of gas phase molecules, the lowest ionization limit of which is such that any one molecule must absorb a minimum of four photons in order to accumulate sufficient excitation energy to achieve collision-free ionization. The probability of a coherent (i.e., simultaneous) four-photon absorption process in any one molecule will generally be very small indeed; MPI will only become significant when the excitation frequency $v$ is tuned so that one (or more) real excited states of the neutral molecule come into resonance at, say, the energy corresponding to two or three photons absorbed. This resonant intermediate level can be regarded as a 'stepping stone' in the over-all ionization process. In Fig. 1, four-photon ionization proceeds via a (relatively) more probable coherent three-photon excitation to a resonant intermediate state, followed by a highly probable (given the high light intensities prevailing in the focal region) one-photon ionization step.

An example of such a $3+1$ REMPI spectrum involving the ethyne molecule $\left(\mathrm{C}_{2} \mathrm{H}_{2}\right)$ is included in Fig. 1. The upper, experimental, spectrum was obtained by focusing the output of a tunable dye laser operating at about $375 \mathrm{~nm}$ into a static room temperature sample of $\mathrm{C}_{2} \mathrm{H}_{2}$ vapour and measuring the resulting total ion current as a function of the excitation wavelength. ${ }^{4}$ Spectroscopic analysis unambiguously identifies the resonant excited state of the neutral molecule as having ${ }^{1} \phi_{u}$ symmetry, and energetic considerations point to it being a Rydberg state arising from the electronic promotion $\pi_{\mathrm{u}}{ }^{3} 3 \mathrm{~d} \sigma_{\mathrm{g}} \leftarrow \pi_{\mathrm{u}}{ }^{4}$. The lower spectrum shows the calculated form of this ${ }^{1} \phi_{\mathrm{u}}-{ }^{1} \Sigma^{+}{ }_{\mathrm{g}}$ three-photon vibronic transition in ethyne; this calculation assumes appropriate values for the ground and excited state rotational constants and for the three-photon rotational linestrengths, ${ }^{4}$ the necessary $3: 1$ weighting in favour of transitions originating from rotational levels with odd $J^{\prime \prime}$ quantum number (reflecting the nuclear spin statistics associated with the two equivalent $I=\frac{1}{2} \mathrm{H}$ nuclei) and a sample temperature of $300 \mathrm{~K}$. The quantitative agreement between the experimental $3+1$ REMPI spectrum and that calculated for just the initial, rate limiting, multiphoton excitation step in the neutral molecule found here and in many other instances, has encouraged the general assumption that it is valid to 

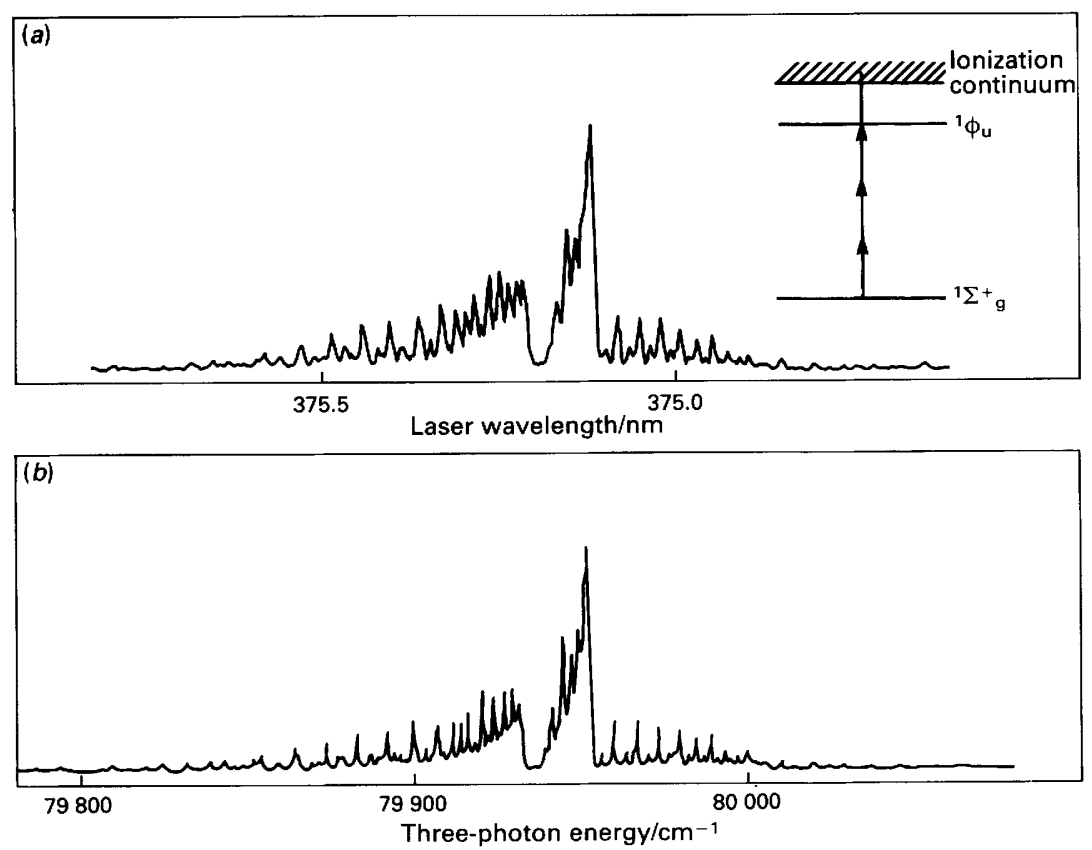

Fig. 1 (a) Three-photon resonant (over-all four-photon ionization) spectrum of 5 Torr $\mathrm{C}_{2} \mathrm{H}_{2}$ in the wavelength region $374.5-376 \mathrm{~nm}$. (b) Simulation of this spectrum, which assumes the electronic transition within the neutral molecule to be of the type ${ }^{1} \phi_{\mathrm{u}}-{ }^{1} \Sigma^{+}{ }_{\mathrm{g}}$, the appropriate spectroscopic parameters for the initial and final states and a sample temperature of $300 \mathrm{~K}$. The good agreement between experiment and theory in this instance implies that there is no marked wavelength or level dependence to the probability of the final one-photon ionization step from the ${ }^{1} \phi_{\mathrm{u}}$ statc

neglect any possible wavelength and/or level dependence to the efficiency of the final photoionization (detection) step. Such assumptions should be adopted with caution; rovibronic level dependent predissociation in the resonant excited state, ${ }^{2}$ alignment of the resonant intermediate state molecules, ${ }^{5,6}$ autoionizing resonances above the ionization limit, ion fragmentation (in experiments employing mass-selective ion detection) and, finally, competition from (unintended) third harmonic generation within the gas sample under investigation ${ }^{7,8}$ (see below) can all cause the experimentally observed $n+1$ REMPI spectrum to have an appearance very different from that predicted simply by application of the appropriate $n$ photon linestrength theory.

Notwithstanding these cautionary comments, REMPI spectroscopy is rightly finding increasingly widespread use as an unobtrusive, spatially localized, detection method for a wide range of gas phase species (including free radicals ${ }^{3}$ ). The technique offers extreme sensitivity and species selectivity (with respect to excitation wavelength, ion mass spectrum and photoelectron kinetic energy).

\section{In Situ Third Harmonic Generation}

Now we introduce the idea of in situ third harmonic generation, in the medium of interest, as another potentially very sensitive and selective detection method. Third harmonic generation (THG) or frequency tripling is, as its name implies, a process whereby radiation of frequency $3 v$ can be generated simply by focusing an intense pulse of laser light (of frequency $v$ ) into a suitable gaseous medium. One important criterion for suitability is that the refractive index of the gaseous medium at the frequency $3 v$ must be smaller than it is at the fundamental frequency $v$. Such anomalous (or negative) dispersion will often be found for a narrow range of $3 v$ frequencies just to the high frequency side of a strong one-photon absorption feature. Notable examples of suitable non-linear media are the rare gases (each of which shows several regions of negative dispersion), and THG in $\mathrm{Ar}, \mathrm{Kr}$ and $\mathrm{Xe}$ have each found use as convenient methods of generating useful intensities of narrow bandwidth, tunable, coherent vacuum ultraviolet (VUV) radiation for use in subsequent spectroscopic or photoionization studies. ${ }^{9,10}$ More recent studies ${ }^{1,7,8}$ have demonstrated the generality of the THG process, in molecular and atomic gases. Consequently, it is always prudent to check for unintended spectral contributions attributable to THG whenever attempting a coherent three-photon excitation between two states which can also be linked by an electric dipole allowed one-photon transition. We note that this last caveat ensures that the ethyne MPI spectrum shown in Fig. 1 is free from any such complications because the ${ }^{1} \phi_{\mathrm{u}^{-}}{ }^{1} \Sigma^{+} \mathrm{g}$ transition, although three-photon allowed, is one-photon forbidden and hence makes no contribution to the VUV absorption spectrum.

In some instances, however, it seems likely that it will prove possible to use in situ THG to advantage, i.e., as an unobtrusive and remote method for detecting spatially and/or temporally localized concentrations of strongly absorbing species. Recent studies concerned with $\mathrm{H}$ atom detection in, respectively, a flowing afterglow, ${ }^{11}$ a d.c. plasma $^{12}$ and in the oscillatory combustion of $\mathrm{H}_{2}{ }^{13}$ provide support for this idea. Traditional detection methods for atomic hydrogen have generally relied on resonance absorption or fluorescence on the very intense $2 \mathrm{p} \leftarrow 1$ s Lyman- $\alpha$ transition $(f \approx 0.4)$; more recently, there have been reports of $\mathrm{H}$ atom detection by $1+1$, $2+1$ and $3+1$ MPI spectroscopy with, in almost all instances, the $n=2$ state providing the resonance enhancement. However, careful inspection of the wavelength dependence of the ion yields observed in the ' $3+1$ MPI' experiments ${ }^{11,13}$ shows the signals to be broadened and blue-shifted from the true Lyman- $\alpha$ frequency; further, the signal maximum is observed to shift to higher frequency as the $\mathrm{H}$ atom concentration is increased. Such observations are inconsistent with the previously outlined picture of the MPI process in which ion formation is enhanced by a discrete resonant intermediate state (here the $2 p$ state). However, as Fig. 2 shows, they are entirely compatible with the idea that the ions result from a simultaneous two-photon absorption process, in which the first photon is formed by THG in the focal volume within the $\mathrm{H}$ atom containing medium itself. The form of the observed ion signal therefore reflects the wavelength dependence of the efficiency for forming VUV radiation by THG [Fig. 2(a)]; this can be 


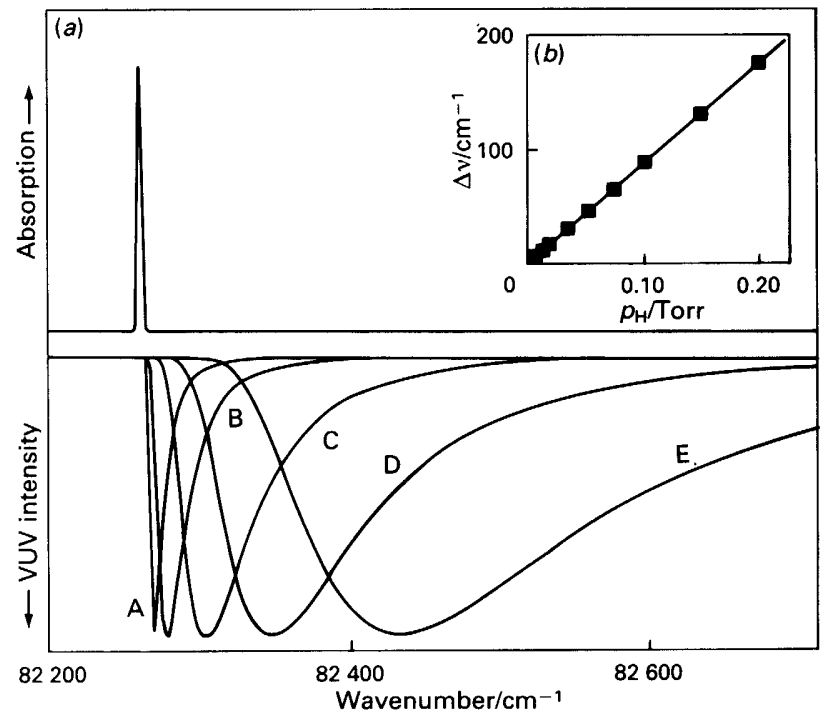

Fig. 2 (a) Calculated form of the excitation profiles for forming VUV radiation by THG on the high frequency side of the $2 p \leftarrow 1$ s Lyman- $\alpha$ transition assuming $\mathrm{H}$ atom concentrations of: $\mathrm{A}, 0.01 ; \mathrm{B}, 0.02 ; \mathrm{C}$, $0.05 ; \mathrm{D}, 0.10$; and $\mathrm{E}, 0.20$ Torr, a single mode Gaussian laser beam and a confocal parameter, $b=2 \mathrm{~mm}$. (b) Plot of $\Delta v$, the frequency difference between the Lyman- $\alpha$ transition and the peak of the calculated VUV excitation profile, against $\mathrm{H}$ atom concentration

confirmed $^{12}$ by direct observation of the generated VUV radiation if the medium itself is sufficiently transmissive at the relevant wavelengths. From the analytical viewpoint, perhaps the most important finding from these recent studies is that, in favourable situations [as Fig. 2(b) shows], the frequency shift of the maximum in the THG excitation profile away from the
Lyman- $\alpha$ transition will scale linearly with the instantaneous $\mathrm{H}$ atom concentration. ${ }^{14,15}$

I am most grateful to all colleagues and students (past and present) at Bristol for their invaluable contributions to all of our endeavours in the area of multiphoton spectroscopy, to Dr. J. E. Butler and colleagues at the Naval Research Laboratory, Washington, DC, and Professor D. L. Baulch and colleagues at Leads University for sharing results prior to publication, and to the Science and Engineering Research Council for their support of our work.

\section{References}

1 For a recent review see Ashfold, M. N. R., and Prince, J. D., Contemp. Phys., 1988, 29, 125.

2 Ashfold, M. N. R., Mol. Phys., 1986, 58, 1.

3 Hudgens, J. W., in Advances in Multiphoton Processes and Spectroscopy, ed. Lin, S. H., World Scientific, Singapore, 1988, vol. 4, p. 171

4 Ashfold, M. N. R., Dixon, R. N., Prince, J. D., and Tutcher, B., Mol. Phys., 1985, 56, 1185.

5 Jacobs, D. C., and Zare, R. N. J. Chem. Phys., 1986, 85, 5457.

6 Jacobs, D. C., Madix, R. J., and Zare, R. N., J. Chem. Phys., $1986,85,5469$.

7 Ashfold, M. N. R., Heryet, C. D., Prince, J. D., and Tutcher, B., Chem. Phys. Lett., 1986, 131, 291.

8 Ashfold, M. N. R., and Prince, J. D., Mol. Phys., 1991, 73, 297.

9 Wallace, S. C., Adv. Chem. Phys., 1981, 47, 153.

10 Hilbig, R., Hilber, G., Lago, A., Wolff, B., and Wallenstein, R., Comments At. Mol. Phys., 1986, 18, 157.

11 Baravian, G., Jolly, J., Persuy, P., and Sultan, G., Chem. Phys. Lett., 1989, 159, 361.

12 Celii, F. G., Thorsheim, H. R., and Butler, J. E., Appl. Phys. Lett., submitted for publication.

13 Baulch, D. L., Griffiths, J. F., and Richter, R., Proc. R. Soc London, in the press.

14 Payne, M. G., and Garrett, W. R., Phys. Rev. A, 1983, 28, 3409.

15 Ashfold, M. N. R., unpublished results.

\title{
Picosecond Photodissociation and Geminate Radical Recombination of Tetraphenylhydrazine
}

\author{
M. G. Hyde and G. S. Beddard \\ Department of Chemistry, University of Manchester, Manchester M13 9PL
}

Cage or geminate recombination has been studied for many years ${ }^{1}$ but only relatively recently has the process been observed on the picosecond time scale. ${ }^{2-4}$ Iodine has been widely studied ${ }^{2,3}$ but vibrational excitation in the ground state after recombination complicates the kinetics.

Lewis and Lipkin ${ }^{5}$ observed that on exposure to ultraviolet (UV) light, tetraphenylhydrazine (TPH) photodissociated into two diphenylaminyl (DPA) radicals. The scheme is

$$
\mathrm{TPH} \stackrel{h v}{\longrightarrow} \text { TPH }^{*} \longrightarrow \underset{\left(\begin{array}{c}
\text { caged pair } \\
\downarrow \\
\downarrow \\
\text { TPH }
\end{array}\right.}{\longrightarrow} \text { 2DPA* }
$$

The absorption spectrum of TPH has transitions in the UV. The DPA radicals also have UV absorption similar to TPH, and additionally, but with lower intensity, bands at $400-500 \mathrm{~nm}$ and slightly more strongly at $750-850 \mathrm{~nm}$.

Picosecond pump and probe experiments ${ }^{6}$ carried out at
$759 \mathrm{~nm}$ show solvent viscosity dependent cage recombination kinetics after excitation at $288 \mathrm{~nm}$ with $1 \mathrm{ps}$ fwhm pulses. The relative molar absorptivity of DPA, $\Delta \varepsilon(t)$, decays over a few tens of picoseconds before reaching a constant level. The rate of initial decay is viscosity dependent and is due to cage recombination to reform TPH, most probably in the ground state, as shown above. An instrument limited, solvent independent, rise-time of the molar absorptivity of DPA was observed; hence the TPH excited singlet state $\left(S_{1}\right)$ lifetime must be approximately $1 \mathrm{ps}$. This is faster than, but still consistent with, a value of $\leqslant 1.8$ ps calculated from the fluorescence quantum yield and application of the Strickler-Berg equation and implies that the rate of energy redistribution in $S_{1}$ must be $\geqslant 10^{12} \mathrm{~s}^{-1}$ as the $\pi \pi^{*}$ absorption is excited but the $\mathrm{N}-\mathrm{N}$ bond broken.

Models of geminate recombination assume that the constituents of the reactive pair are initially separated, then undergo random Brownian motion until they either recombine or separate. The probability of reactive pair survival, $p(\tau)$, has 
been calculated by Scheider $r^{7}$ using diffusion theory and by Shin and $\mathrm{Kapral}^{8}$ using a kinetic approach giving

$$
\begin{aligned}
p(t)=1 & -\frac{\lambda}{\kappa(\lambda+1)}\left\{\operatorname{erfc}\left[\frac{\kappa-1}{2 \tau^{\frac{1}{2}}}\right]\right. \\
& -\exp [(1+\lambda)(\kappa-1)] \times \exp \left[(1+\lambda)^{2} \tau\right] \\
& \left.\times \operatorname{erfc}\left[(1+\lambda) \tau^{\frac{1}{2}}+(\kappa-1) / 2 \tau^{\frac{1}{2}}\right]\right\}
\end{aligned}
$$

The measured molar absorptivity of DPA, $\Delta \varepsilon(t)$, is directly proportional to $p(\tau)$. The parameter $\lambda$ is given by $\lambda=k_{\mathrm{f}} / k_{\mathrm{d}}$, with $k_{\mathrm{d}}=4 \pi \sigma D$ the rate constant for diffusion and $k_{\mathrm{f}}=4 \pi \sigma^{2} v$ the reaction rate. The reduced time $\tau$ is $\tau=t / \tau_{0}$, with $\tau_{0}=\sigma^{2} / D$, and $\kappa$ is the reduced separation $\kappa=r_{0} / \sigma$. The diffusion coefficient is $D, \sigma$ is the reaction radius and the velocity is $v$.

As there are three parameters $\left(\tau_{0}, \kappa\right.$ and $\left.\lambda\right)$ describing eqn. (1), transient decays can be fitted by a wide range of values. In order to obtain consistent fitting with different solvents, we used two guessed values of $\sigma$ and estimated $\tau_{0}$ from $D$. The slip limit is used to calculate $D=k_{\mathrm{B}} T / 4 \pi \eta d ; \eta$ is the viscosity and the diffusion radius, $d=2.43 \AA$, is calculated from the molecular volume which is estimated assuming a spherical DPA. For $\sigma=2$ and $3 \AA, \tau_{0}$ has values of 30 and $67.7 \mathrm{ps,}$ respectively, for $\eta=1.02 \mathrm{cP}$. Similar values for $\mathrm{K}$ and $\lambda$ were obtained across the ten solvents used, hexane to hexadecane, $\eta$ $=0.326-3.34 \mathrm{cP}$. Only average values over the range of solvents are calculated, which are: $\sigma=2 \AA,\langle\kappa\rangle^{*}=1.38$ (standard error 0.056) and $\langle\lambda\rangle=1.30(0.051)$; and $\sigma=3 \AA$, $\langle\kappa\rangle=1.17(0.047)$ and $\langle\lambda\rangle=0.89(0.074)$. From these values of $\mathrm{K}$ the initial separation, $r_{0}$, is 2.76 and $4.14 \AA$ for $\sigma=2$ and $3 \AA$, respectively, which agrees with separations measured from ESR experiments. ${ }^{9}$ With a value for $\lambda$ and using diffusion theory to calculate $k_{\mathrm{d}}, k_{\mathrm{f}}$ can be estimated. With $\eta=1 \mathrm{cP}$ and in the slip limit, $k_{\mathrm{d}}=9.8 \times 10^{9} \mathrm{dm}^{3} \mathrm{~mol}^{-1} \mathrm{~s}^{-1}$ and hence $k_{\mathrm{f}}$ is in the range from $10.0 \times 10^{9}$ to $8.7 \times 10^{9} \mathrm{dm}^{3} \mathrm{~mol}^{-1} \mathrm{~s}^{-1}$.

The assumptions underlying the derivation of eqn. (1) should not necessarily apply to molecules such as TPH, which are of a size similar to that of the solvent molecules. In formulating eqn. (1), the species are assumed to move apart translationally, but on the short timescale this should be prevented to any great extent by collisions with the surrounding solvent; further, the solvent will have to be displaced and enter the space between the two radicals to prevent immediate recombination. We propose an alternative simple model to explain our data, which is that rotational motion of the DPA radicals will initially separate the $\mathrm{N}$ atoms more rapidly than

\footnotetext{
* Figures in angle brackets are average values.
}

translation. This can involve little or no solvent displacement and can therefore be rapid, preventing immediate recombination. Eventually, translational motion must also separate the radicals but we propose that this is initially a smaller effect. This hypothesis is supported by the observation that the initial decay of relative molar absorptivity is directly proportional to the solvent viscosity with a slope of $60 \AA^{3}$ which is comparable to the volume calculated from space filling molecular models of 65-75. $\AA^{3}$.

We have not been able to solve the diffusion equations for two spheres rotating and recombining as described above. The solution for one moving and one fixed sphere has been obtained analytically and gives $p(t)$ decays similar to that from eqn. (1). This corresponds to the situation of a rotating particle dissociating and recombining from a large fixed molecule, or of an atom dissociating from a molecule where, because of symmetry, rotation of the atom is not important. A randomwalk model has also been used for the two sphere diffusion and slower decays of $p(t)$ are obtained than with one fixed sphere and in better agreement with experiment. These results will be described elsewhere. ${ }^{10}$

The recombination can also be viewed in another way. As the slow solvent dynamics temporarily prevent separation of the radicals, they are effectively trapped in a shallow potential well. In this instance the recombination is not dissimilar to that of crossing a potential energy barrier, and hence Kramers' equation can be applied. ${ }^{11}$ This describes the barrier crossing as an activated process, as in the Arrhenius equation but with the pre-exponential term modified by the barrier and well frequencies and solvent dynamics. The TPH data are well described by this equation. A fuller account of this work will be published elsewhere. ${ }^{12}$

\section{References}

1 Rabinowich, E., and Wood, W. C., Trans. Faraday Soc., 1936, 32, 547

2 Chang, T. J., Hoffman, G. W., and Eisenthal, K. B., Chem. Phys. Lett., 1974, 25, 201.

3 Smith, D. E. and Harris, C. B., J. Chem. Phys, , 1987, 87, 2709.

4 Scott, T. W., and Liu, S. N., J. Phys. Chem., 1989, 93, 1393.

5 Lewis, G. N., and Lipkin, D., J. Am. Chem. Soc., 1942, 64, 2801

6 Harrison, R. J., Pearce, B., Beddard, G. S., Cowan, J., and Sanders, J., Chem. Phys., 1987, 116, 2680

7 Scheider, W., J. Phys. Chem., 1972, 76, 349.

8 Shin, K. S., and Kapral, R., J. Chem. Phys., 1978, 69, 3685.

9 Wiersma, D. A., and Kommandeur, J., J. Chem. Phys., 1969, 50, 2794.

10 Beddard, G. S., and Masters, A. J., in preparation.

11 Bowman, R., Millar, D., and Eisenthal, K. B., J. Chem. Phys., $1988,89,762$.

12 Hyde, M., and Beddard, G. S., Chem. Phys., 1991, in the press 